\title{
LOCALIZING GROUPS WITH ACTION
}

\author{
GEORG PESCHKE
}

\begin{abstract}
When localizing the semidirect product of two groups, the effect on the factors is made explicit. As an application in Topology, we show that the loop space of a based connected $C W$-complex is a $P$-local group, up to homotopy, if and only if $\pi_{1} X$ and the free homotopy groups $\left[S^{k-1}, \Omega X\right]$, $k \geq 2$, are $P$-local.
\end{abstract}

\section{Introduction}

The study of groups $G$ in which the functions $\rho_{p}: G \rightarrow G, \rho_{p}(g)=g^{p}$, are, for certain primes $p$, bijective, has a long history; see Malcev [9], Batumslag [1] and the references there. After Sullivan [15], Bousfield-Kan [3], Hilton [5] and Hilton-Mislin-Roitberg [8] this study appears now in the guise of localizing a group with respect to a given set of primes $P$. In a $P$-local group the functions $\rho_{n}$ are bijective if $n$ belongs to the multiplicative closure of the set of primes $P^{\prime}$, which is complementary to $P$.

According to Ribenboim [11], there is a $P$-localizing functor from the category of groups to the category of $P$-local groups, $\mathcal{G} \rightarrow \mathcal{G} p$. While the properties of this functor, when restricted to the category of nilpotent groups, are well understood (see [5] and [7]), its properties in general are not clear at all.

For example, on nilpotent groups the $P$-localizing functor is exact, but not in general. E.g., the exact sequence $Z / 3 \rightarrow S_{3} \rightarrow Z / 2$ for the symmetric group of 3 elements gets sent to $Z / 3 \rightarrow 0 \rightarrow 0$, when localizing at $3 . S_{3}$ is a semidirect product $Z / 3 \rtimes Z / 2$ and the purpose of this paper is to investigate the effect of localization on semidirect products $G=H \times R$ :

Since localization is functorial, $G p$ is again a semidirect product $G p \cong K \times R p$. Therefore, it is desirable to understand the relation between $H$ and $K$. We will discover that $K$ is the $P$-localization of $H$ with respect to the change of operator groups from $R$ to $R p$.

To explain this, we use the category ${ }_{R} \mathcal{G}$ of $R$-groups (i.e. groups on which the group $R$ acts on the left) and $R$-homomorphisms (i.e. group homomorphisms $f: H \rightarrow H^{\prime}$ with $f(r \cdot h)=r \cdot f(h)$ for all $h \in H$ and $r \in R$ ). Further a group 
homomorphism $\gamma: R \rightarrow S$ induces the change-of-operator-groups functor $\gamma^{*}: S \mathcal{G} \rightarrow{ }_{R} \mathcal{G}$. For $H \in{ }_{R} \mathcal{G}, K \in S \mathcal{G}$, a group homomorphism $f: H \rightarrow K$ is a $\gamma$-homomorphism, if $f: H \rightarrow \gamma^{*} K$ is an $R$-homomorphism. We then construct a left adjoint $\gamma A d$ for $\gamma^{*}$; see 1.5 .

Now, $s \mathcal{G}$ contains a subcategory $s \mathcal{G} p$ consisting of such groups on which $S$ acts $P$-locally ; see 1.2. Accordingly, we construct a left adjoint ${ }_{S} L p:{ }_{S} \mathcal{G} \rightarrow$ ${ }_{S} \mathcal{G} p$; see 1.6. The composite ${ }_{\gamma} L p:=S^{L} p_{\gamma} A d:{ }_{R} \mathcal{G} \rightarrow{ }_{S} \mathcal{G} p$ is left adjoint to the restriction of $\gamma^{*}$ to $S \mathcal{G} p$. It then follows that $(H \times R) p \cong\left({ }_{e} L p H\right) \rtimes R p$, where $e: R \rightarrow R p P$-localizes.

Remarks.

(1) The functor ${ }_{\gamma} A d$ is of independent interest. For example, let $s A d$ correspond to the unique homomorphism $\{1\} \rightarrow S$. Then $S A d$ provides the foundation for a theory of $S$-groups by generators and relations.

(2) The problem of localizing semidirect products has also been studied by Casacuberta [4] in the case where the normal subgroup $H$ is abelian, and by A. Reynol when $H$ is finite abelian [12].

(3) Our study is also of interest in Topology; see 1.7 and 1.8 .

It is a pleasure to acknowledge several useful conservations with $\mathrm{K}$. Varadarajan. Also I owe insight into the matter to correspondence with $\mathrm{P}$. Hilton and C. Casacuberta.

1. We now take up the announced investigation. So let $R$ be a group acting on another group $H$ via a homomorphism $\phi: R \rightarrow A u t H$. The corresponding semi-direct product is denoted by $H \rtimes_{\phi} R$ or $H \rtimes R$ if there is no risk of confusion.

Lemma 1.1. $G=H \rtimes R$ is $P$-local $\Leftrightarrow$ the following two conditions hold:

(i) $R$ is $P$-local;

(ii) For all $r \in R$ and $n \in P^{\prime}$, the function

$$
\rho_{r, n}: H \rightarrow H, h \mapsto h \phi_{r}(h) \phi_{r^{2}}(h) \ldots \phi_{r^{n-1}}(h)
$$

is a bijection, where $\phi_{r}$ denotes the automorphism $\phi(r)$ of $H$.

Proof: This follows from $(h, r)^{n}=\left(\rho_{r, n}(h), r^{n}\right)$.

The functions $\rho_{r, n}$ have been used already by Baumslag in a setting involving wreath products; see [2].

Definition 1.2. $R$ acts $P$-locally on $H: \Leftrightarrow$ for all $r \in R$ and $n \in P^{i}$, the function $\rho_{r_{,},}$of (1.1) is a bijection.

The notion of a $P$-local action has independently been introduced by Rodicio, [13]. Since $\rho_{1, n}(h)=h^{n}$, if $R$ acts $P$-locally on $H$, then $H$ is $P$-local. We write ${ }_{R} \mathcal{G} p$ for the category of $R$-groups on which $R$ acts $P$-locally.

It is straightforward to prove 
Lemma 1.3. Let

$$
\begin{array}{cccc}
H & \rightarrow H \rtimes_{\phi} R & \rightarrow & R \\
\alpha \downarrow & \beta \downarrow & & \downarrow \\
K & \mapsto K \rtimes_{\psi} S & \rightarrow & S
\end{array}
$$

be a commuting diagram of split exact sequences of groups. Then $\beta P$-localizes in $\mathcal{G}$ if and only if the following three conditions hold:

(i) $\gamma P$-localizes in $\mathcal{G}$;

(ii) $S$ acts $P$-locally on $K$;

(iii) For all $L \in S \mathcal{G}$ on which $S$ acts $P$-locally and every $\gamma$-homomorphism $\nu: H \rightarrow L$, there is a unique $S$-homomorphism $\nu^{3}: K \rightarrow L$, with $\nu=\nu^{\prime} \alpha$.

This suggests

Definition 1.4. Let $H \in{ }_{R} \mathcal{G}, K \in S \mathcal{G}$ and let $\gamma: R \rightarrow S$ be a homomorphism. Then $\alpha: H \rightarrow K P$-localizes with respect to $\gamma$ if and only if the following three conditions hold:

(i) $S$ acts $P$-locally on $K$;

(ii) $\alpha$ is a $\gamma$-homomorphism;

(iii) $\alpha$ satisfies the universal property 1.3 (iii) above

Thus, Lemma (1.3) can be restated as

Lemma 1.3'. $\beta P$-localizes in $\mathcal{G}$ if and only if $\gamma P$-localizes in $\mathcal{G}$ and $\alpha P$ localizes with respect to $\gamma$.

Now let $\gamma: R \rightarrow S$ be given. The construction of a left adjoint functor ${ }_{\gamma} L p:{ }_{R} \mathcal{G} \rightarrow{ }_{S} \mathcal{G} p$ to the composite functor ${ }_{S} \mathcal{G} p \stackrel{\text { inciusion }}{\longrightarrow}{ }_{S} \mathcal{G} \stackrel{\gamma^{*}}{\rightarrow}{ }_{R} \mathcal{G}$ is done in two steps.

Theorem 1.5. $\gamma^{*}:{ }_{S} \mathcal{G} \rightarrow{ }_{R} \mathcal{G}$ has a left adjoint ${ }_{\gamma} A d:{ }_{R} \mathcal{G} \rightarrow{ }_{S} \mathcal{G}$.

Theorem 1.6. The inclusion functor $s \mathcal{G} p \rightarrow s \mathcal{G}$ has a left adjoint left inverse ${ }_{s} L p: s \mathcal{G} \rightarrow s \mathcal{G} p$.

It then follows from 1.3' that $(H \times R) p \cong\left({ }_{e} L p H\right) \rtimes R p$, where $e: R \rightarrow R p$ $P$-localizes.

Here is an interesting application of $P$-local actions in Topology.

Theorem 1.7. Let $X$ be a based connected $C W$-complex. Then, $\pi_{1} X$ and the free homotopy groups $\left[S^{k-1}, \Omega X\right], k \geq 2$, are $P$-local $\Leftrightarrow \Omega X$ is a $P$ local group up to homotopy; i.e. for each $n \in P^{\prime}$, the map $\bar{\rho}_{n}: \Omega X \rightarrow \Omega X$, $\bar{\rho}_{n}(x)=x^{n}$, is a homotopy equivalence.

Proof: " $\Leftarrow$ " Recall that $\bar{\rho}_{n}$ induces $\rho_{n}$ on $\pi_{0} \Omega X$ and on all free homotopy groups $\left[S^{k-1}, \Omega X\right], k \geq 2$. If $\bar{\rho}_{n}$ is a homotopy equivalence, then $\rho_{n}$ is a bijection. Thus $\pi_{0} \Omega X \cong \pi_{1} X$ and $\left[S^{k-1}, \Omega X\right]$ are $P$-local. 
$" \Rightarrow$ "Recall from [10] that $\Omega X$ is an $H$-semidirect product: $\Omega X \simeq(\Omega X)_{0} \rtimes$ $\pi_{1} X$ and, as a consequence, that $\left[S^{k-1}, \Omega X\right] \cong \pi_{k} X \rtimes \pi_{1} X$, for all $k \geq 2$. Since $\pi_{1} X$ is $P$-local, $\bar{\rho}_{n}$ determines a bijection of the connected components of $\Omega X$. Since $(\Omega X)_{0}$ is a simple space, the restriction of $\rho_{n}$ to $(\Omega X)_{0} \times\{r\}, r \in \pi_{I} X$, induces the homomorphism

$$
\begin{aligned}
& \pi_{k-1}(\Omega X)_{0} \times\{r\} \cong\left[S^{k-1},(\Omega X)_{0} \times\{r\}\right] \rightarrow\left[S^{k-1},(\Omega X)_{0} \times\left\{r^{n}\right\}\right] \cong \\
& \cong \pi_{k-1}(\Omega X)_{0} \times\left\{r^{n}\right\}
\end{aligned}
$$

By hypothesis, this is a bijection. Thus, $\bar{\rho}_{n}$ is a homotopy equivalence.

Corollary 1.8. Loop spaces of $P$-local nilpotent $C W$-complexes are $P$-local groups up to homotopy.

Proof: If $X$ is a $P$-local nilpotent space, then $\pi_{1} X$ is $P$-local. Furthermore, the groups $\left[S^{k-1}, \Omega X\right], k \geq 2$, are semidirect products of the $P$-local groups $\pi_{k} X$ and $\pi_{1} X$ with respect to a nilpotent action of $\pi_{1} X$ on $\pi_{k} X$. By a result of Hiton [6], the groups $\left[S^{k-1}, X\right]$ are $P$-local, for $k \geq 2$; compare also Roitberg [14]. Now apply 1.7 .

\section{Proof of Theorem 1.5}

We need the following lemma whose proof is a little tedious but straightforward.

Lemma 2.1. Let $R$ act on $H$ via $\phi: R \rightarrow$ Aut $H$. Let $D:=\left\{r h r^{-1} \phi_{r}\left(h^{-1}\right):\right.$ $r \in R, h \in H\} \subset H * R$. Let $\bar{H}, \bar{D}$ denote the normal closure of $H, D$ in $H * R$. Then $\bar{D}$ is normal in $\vec{H}$ and $\bar{H} / \bar{D}$ is isomorphic to $H$.

Step 1 for the proof of (1.5): Construction of ${ }_{\gamma} A d$

Let $R$ act on $H$ via $\phi: R \rightarrow$ Aut $H$ and consider the diagram

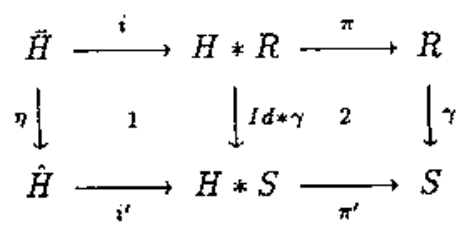

where $\pi, \pi^{\prime}$ are the canonical epimorphisms making square 2 commute. $\bar{H}:=$ ker $\pi$ and $\hat{H}:=\operatorname{ker} \pi^{t}$. Note that $(I d * \gamma)(\bar{H}) \subset \hat{H}$ and let $\eta$ be the restriction of $I d * \gamma$ to $\bar{H}$. Then square 1 also commutes.

By design, $R$ acts on $\bar{H}$ by conjugation and $S$ acts on $\hat{H}$ by conjugation and $\eta$ is a $\gamma$-homomorphism. Using (2.1), we relate these actions to the given action of $R$ on $H$. We have refined the method of $H N N$-extensions. 
Let $D:=\left\{r h r^{-1} \phi_{r}\left(h^{-1}\right): r \in R, h \in H\right\} \subset H * R$. By $(2.1), \bar{D} \subset \bar{H}$. Let $\hat{D}$ be the normal closure of $\eta(D)$ in $H * S$. Since $\eta(D) \subset \hat{H} \triangleleft(H * S), \hat{D} \subset \hat{H}$. Take $K:={ }_{\gamma} A d(H):=\hat{H} / \hat{D}$. Then $\eta$ defines $\alpha: H \cong \hat{H} / \hat{D} \rightarrow \hat{H} / \hat{D}=K$.

The action of $S$ on $\hat{H}$ by conjugation passes down to an action $\psi: S \rightarrow$ Aut $K$. Explicitly, $\psi_{S}(\hat{h} \hat{D})=s \hat{h} s^{-1} \hat{D}$. The action of $R$ on $\bar{H}$ by conjugation passes down to the original action $\phi$, by (2.1). It is clear that $\alpha$ is a $\gamma$-homomorphism.

Step 2. Vertification of the universal property of $\alpha: H \rightarrow K$. Let $S$ act on $L$ via $\theta: S \rightarrow A u t L$. Let $\nu: H \rightarrow L$ be a $\gamma$-homomorphism. Consider the diagram

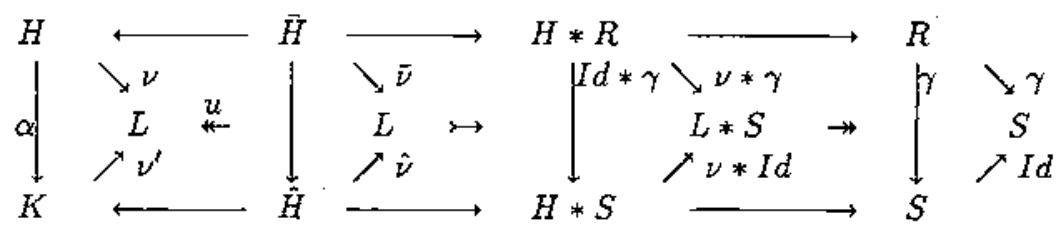

The right hand prism commutes and induces homomorphisms $\bar{\nu}, \hat{\nu}$ by restriction. Further, $\bar{\nu}(D) \subset$ ker $u$. Consequently, $\hat{\nu}(\hat{D}) \subset$ ker $u$, showing that $\hat{\nu}$ factors through $K$ with $\nu^{\prime}: K \rightarrow L$. Since $\dot{\nu}$ is an $S$-homomorphism, so is $\nu^{\prime}$.

It is straightforward to check uniqueness of $\nu^{\prime}$ on the generators $x h x^{-1}$ of $K$, where $x \in H * S, h \in H$. That ${ }_{\gamma} A d$ is a functor is immediate. This completes the proof of (1.5).

It follows directly from the construction of ${ }_{\gamma} A d$ that (2.2): ${ }_{\gamma}$ Ad preserves epimorphisms.

\section{Proof of Theorem 1.6}

Let

$$
\begin{aligned}
& { }_{s} \mathcal{U} p:=\left\{K \in{ }_{s} \mathcal{G}: \rho_{s, n} \text { is } 1-1 \text { for all } s \in S, n \in P^{\prime}\right\} \\
& { }_{s} \mathcal{E} p:=\left\{K \in{ }_{s} \mathcal{G}: \rho_{s, n} \text { is onto for all } s \in S, n \in P^{\prime}\right\}
\end{aligned}
$$

Then ${ }_{s} \mathcal{G} p:=s_{\mathcal{U}} \cap_{s} \mathcal{E}_{p}$ is the category of $S$-groups on which $S$ acts $P$ locally.

We construct functors $s \sqrt{ }_{p}: s \mathcal{G} \rightarrow s \mathcal{G}$, which create preimages for the functions $\rho_{s, n}$ as well as ${ }_{s} U p: s \mathcal{G} \rightarrow{ }_{s} \mathcal{U} p$, which make preimages of the functions $\rho_{s, n}$ unique.

Let $S$ act on $K$ via $\psi: S \rightarrow$ AutK. Let $F K$ denote the free group with basis $\left\{k_{s, n}: k \in K, s \in S, n \in P^{\prime}\right\}$ and let $\xi K:=s A d F K$ denote the free $S$-group with that basis. If $\theta: S \rightarrow A u t \xi K$ denotes the corresponding $S-$ action, then $S$ acts on $K * \xi K$ by $S \ni s \mapsto \psi_{s} * \theta_{s} \in A u t(K * \xi K)$. Let $N$ denote the 
$S$-invariant normal closure of the set $\left\{\rho_{s, n}\left(k_{s, n}\right) k^{-1}: k \in K, s \in S, n \in P^{\prime}\right\}$ in $K * \xi K$.

Definition 3.1. $s \sqrt{ }_{p} K:=K * \xi K / N$.

There is a canonical homomorphism $t: K \rightarrow s \sqrt{p}_{p} K$. By design, im $t \subset$ im $\rho_{s, n}$, for all $s \in S$ and $n \in P^{\prime}$. Further, an $S$-homomorphism $f: K \rightarrow K^{\prime}$ induces the $S$-homomorphism $\xi f: \xi K \rightarrow \xi K^{\prime}$ via the function $k_{s, n} \rightarrow[f(k)]_{s, n}$ on bases. Hence, the $S$-homomorphism $(f * \xi f): K * \xi K \rightarrow K^{\prime} * \xi K^{\prime}$ is defined. Passing to quotients, it yields the $S$-homomorphism $s \sqrt{p} f: s \sqrt{p} K \rightarrow s \sqrt{p} K^{\prime}$.

\section{Lemma 3.2.}

(i) $s \sqrt{p}: s \mathcal{G} \rightarrow s \mathcal{G}$ is a covariant functor.

(ii) $S \sqrt{ } p$ preserves epimorphisms.

(iii) The homomorphism $t: K \rightarrow s \sqrt{p} K$ defines a natural transformation of the identity functor on $S \mathcal{G}$ to $S \sqrt{ } p$.

(iv) If $f: K \rightarrow L$ is an $S$-homomorphism such that $\rho_{\ell, n}$ is $(1-1)$ and onto im $f$ for all $\ell \in L$ and $n \in P^{i}$, then there is a unique $S$-homomorphism $f^{\prime}: s \sqrt{ }{ }_{p} K \rightarrow L$ with $f=f^{\prime} t$.

Proof: (i), (ii), (iii) are straightforward from the construction.

(iv) The universal property of $S A d$ yields a unique $S$-homomorphism $d$ : $\xi K \rightarrow L$ corresponding to the homomorphism $F K \rightarrow L, k_{s, n} \mapsto \rho_{s, n}^{-1} f(k)$. Observe that $\operatorname{ker}\left(K * \xi K \rightarrow s \sqrt{ }{ }_{p} K\right) \subset \operatorname{ker}(f * d)$. Hence, $f^{\prime}$ exists. Uniqueness of $f^{\prime}$ follows from $f^{\prime \prime} \rho_{s, n}=\rho_{s, n} f^{\prime \prime}$, for any $f^{\prime \prime}: s \sqrt{ }{ }_{p} K \rightarrow L$ with $f=f^{\prime \prime} t$.

Definition 3.3. Let $K$ be any $S$-group.

$$
{ }_{s} E p K:=\lim _{\rightarrow} K \rightarrow s \sqrt{p} K \rightarrow\left(s \sqrt{p}_{p}\right)^{2} K \rightarrow \ldots
$$

By induction, using Lemma 3.2 , we get

\section{Proposition 3.4.}

(1) (i) ${ }_{s} E_{p}:{ }_{s} \mathcal{G} \rightarrow{ }_{s} \mathcal{E} p$ is a covariant functor.

(2) (ii) ${ }_{s} E_{p}$ preserves epimorphisms.

(3) (iri) The canonical homomorphism $\tau: K \rightarrow{ }_{s} E p K$ defines a natural transformation of the identity functor on $s \mathcal{G}$ to $s E_{p}$.

(4) (iv) If $f: K \rightarrow L$ is an $S$-homomorphism and $S$ acts $P$-locally on $L$, then there is a unique $S$-homomorphism $f^{\prime}: s E p K \rightarrow L$ with $f=f^{\prime} \tau$.

To make the functions $\rho_{s, n}$ of an $S$-group $K(1-1)$, we factor out a suitable subgroup. Let

$$
s^{a p K}:=\cap\left\{\operatorname{ker}(f: K \rightarrow U): U \in s^{\mathcal{U}} p, f \text { any } S \text {-homomorphism }\right\} .
$$


Definition 3.5. ${ }_{s} U p K:=K / s a p K$.

It follows that ${ }_{s} U p K \in{ }_{s} \mathcal{U}$. Further, if $f: K \rightarrow K^{\prime}$ is an $S$-homomorphism, then $f\left(s_{s} a p K\right) \subset s a p K^{\prime}$. So $f$ induces ${ }_{s} U p f: s U p K \rightarrow{ }_{s} U p K^{t}$.

The lemma below is a direct consequence of this definition.

Lemma 3.6.

(i) $s U p: s \mathcal{G} \rightarrow s \mathcal{U}$ is a covariant functor.

(ii) The canonical epimorphism $\sigma: K \rightarrow{ }_{s} U p K$ defines a natural tranformation of the identity functor on ${ }_{S} \mathcal{G}$ to ${ }_{S} U p$.

(iii) ${ }_{S} U p$ preserves epimorphisms.

(iv) If $f: K \rightarrow L$ is an $S$-homomorphism and $L \in{ }_{s} U p$, then there is a unique homomorphism $f^{\prime}: s U p K \rightarrow L$ with $f=f^{\prime} \sigma . s U p$ is left adjoint left inverse to the inclusion functor $s \mathcal{U} p \rightarrow s \mathcal{G}$.

Definition 3.7: Let $\gamma: R \rightarrow S$ be a group homomorphism. Let ${ }_{\gamma} L p:=$ ${ }_{S} U_{p} E_{p_{\gamma}} A d: \mathcal{G}_{R} \rightarrow{ }_{S} \mathcal{G} p$ be the composite of the three functors.

Note that the natural transformations associated with ${ }_{s} U p,{ }_{s} E p, \gamma A d$ define a natural transformation $e\left(=_{\gamma} e p\right)$ of the identity functor on ${ }_{R} \mathcal{G}$ to ${ }_{S} L p$.

Proposition 3.8. Let $\gamma: R \rightarrow S$ be a group homomorphism.

(i) ${ }_{S} L p:{ }_{R} \mathcal{G} \rightarrow{ }_{S} \mathcal{G} p$ is a covariant functor which is left adjoint to the change-of-operator-groups functor $\gamma^{*}: s \mathcal{G} p \rightarrow R \mathcal{G}$.

(ii) ${ }_{s} L p$ preserves epimorphisms.

Proof: Combine 1.5, 2.2, 3.4, 3.6.

This completes the proof of 1.6 .

The author is grateful to the referee for pointing out the connection of [2], $[6],[13]$ with the present paper.

\section{References}

1. G. BaUmSlaG, Some aspects of groups with unique roots, Acta Math. $104(1960), 217-303$.

2. G. BAumslaG, "Roots and wreath products," Proc. Cam. Phil. Soc. 56, 1960, pp. 109-117.

3. A.D.K. Bousfield and D.W. Kan, "Homotopy Limits," Completions and Localizations, Springer Lect. Notes 304, 1972.

4. C. CASACUBERTA, "Espais topologics amb accions P-locals," $\mathrm{Ph}$. D. Thesis, Universitat Autònoma de Barcelona, 1988.

5. P. Hilton, "Localization and cohomology of nilpotent groups," Math. Z.132, 1973, pp. 265-286. 
6. P. HILTON, Nilpotent actions on nilpotent groups, Springer Lect. Notes 450 (1975), 174-196.

7. P. Huton, "Nilpotente Gruppen und nilpotente Raeume," Springer Lec. Notes, 1053, 1984.

8. P. HILTON, G. MISLIN AND J. ROITBERG, "Localization of nilpotent groups and spaces," North Holland Math. Studies, 15, 1975.

9. A.I. MALCEV, "Nilpotent torsion free groups," Izv. Akad. Nauk. SSSR Ser. Mat. 13, Russian, 1949, pp. 202-212.

10. G. PESCHKE, $H$-semidirect products, Can. Math. Bull. 30 (1987), 402-411.

11. P. RIBENBOIM, Equations in groups with special emphasis on localization and torsion, 1 and 2 , preprint.

12. A. REYNOL, $P$-localization of some classes of groups, preprint.

13. A.G. Rodicio, "Métodos homológicos en grapos P-locales," Ph.D. thesis, 1986.

14. J. RoItberg, Note on nilpotent spaces and localization, Math. Z. 137 (1974), 67-74.

15. D. Sullivan, "Genetics of homotopy theory and the Adams conjecture," Ann. Math. 100, 1974, pp. 1-79.

Dept. Mathematics

Faculty of Science

University of Alberta

632 Central Academic Building

Edmonton, CANADA T6G 2G1

Rebut el 11 d'Octubre de 1988 